\title{
Towards a complete in-silico assessment of the outcome of cochlear implantation surgery
}

\author{
Nerea Mangado ${ }^{1}$, Mario Ceresa ${ }^{1}$, Heval Benav ${ }^{2}$, Pavel Mistrik ${ }^{2}$, Gemma Piella ${ }^{1}$, \\ and Miguel A. González Ballester ${ }^{1,3}$ \\ 1 SIMBioSys group, Department of Information and Communication Technologies, \\ Universitat Pompeu Fabra, Barcelona, Spain \\ 2 Med-EL, Innsbruck, Austria \\ 3 ICREA, Barcelona, Spain
}

Address of correspondence: Miguel A. González Ballester, Department of Information and Communication Technologies, Universitat Pompeu Fabra, C/ Roc Boronat 138, Barcelona 08018, Spain. Email: ma.gonzalez@upf.edu

\begin{abstract}
Cochlear implantation (CI) surgery is a very successful technique, performed on more than 300.000 people worldwide. However, since the challenge resides in obtaining an accurate surgical planning, computational models are considered to provide such accurate tools. They allow us to plan and simulate beforehand surgical procedures in order to maximally optimize surgery outcomes, and consequently provide valuable information to guide pre-operative decisions. The aim of this work is to develop and validate computational tools to completely assess the patient-specific functional outcome of the CI surgery. A complete automatic framework was developed to create and assess computationally CI models, focusing on the neural response of the auditory nerve fibers (ANF) induced by the electrical stimulation of the implant. The framework was applied to evaluate the effects of ANF degeneration and electrode intra-cochlear position on nerve activation. Results indicate that the intra-cochlear positioning of the electrode has a strong effect on the global performance of the CI. Lateral insertion provides better neural responses in case of peripheral process degeneration, and it is recommended, together with optimized intensity levels, in order to preserve the internal structures. Overall, the developed automatic framework provides an insight into the global performance of the implant in a patient-specific way. This enables to further optimize the functional performance and helps to select the best CI configuration and treatment strategy for a given patient.
\end{abstract}

Keywords: cochlear implant, computational model, finite element, electrical simulation, neural response. 


\section{Introduction}

Cochlear implantation (CI) has proven to be a successful procedure to restore hearing in patients who suffer from medium to severe hearing loss. Nevertheless, the level of restoration highly depends on patient-specific factors, such as the patient's anatomy [1-3], making the prediction of CI performance a challenging process. Currently, statistical relations based on the patient's deafness duration and residual hearing are mainly used to estimate the expected implant performance. Some authors have tried to identify other parameters that may affect the level of restoration and, thus, could be established as predictors of the implant outcomes [2-4]. Nevertheless, a clear association between pre-operative measurements and the patient's CI outcome has not yet been observed, since a high number of factors, including pre-operative and implant information, contribute to a high variability on the implant performance [2]. Previous studies proposed the brain metabolic activity as a factor to effectively predict CI results before surgery [4, 5]. However, such clinical pre-operative data is not always available, and these hypotheses still need to be fully tested with further functional experiments [4]. Consequently, new prediction tools can play an important role in the implant performance assessment. The development of advanced computational tools can provide an estimation of the patient-specific CI outcome to directly support pre-operative decisions.

Although in-silico studies have not been commonly applied into the clinical practice of CI, they have shown their potential to become valuable tools to compute predictive performance of the implantable device [6-9]. In the past, some authors assessed the resulting electric field accounting for different cochlear implant set-ups, such as stimulation protocols [10-12], or neural fiber conditions, contrasting both intact and degenerated fibers [8, 13]. Other authors focused their studies to improve finite element models by including the ear canal and middle ear [14] or the whole human head [15, 16], and to evaluate the neural excitation patterns $[6,17,18]$. However, these studies did not lead to a complete and automatic computational approach encompassing all stages, from the generation of a highly detailed patient-specific model of the cochlear anatomy to the neural response evaluation. Some previous studies considered volume conduction models from simplified [7, 16] and parametric [9] representations of the cochlear anatomy, built from guinea pig cochleae [19], or considered a more detailed geometrical model [20]. However, simplified or generic models limit the insight on CI performance, due to their high dependence on patient-specific factors, pointing out to the need for personalized detailed models [20].

The neural response of the auditory nerve fibers (ANF) to a CI stimulus has been paid special attention during the last decades [17, 18, 21, 22]. Dynamic range, place pitch or spread of excitation on the auditory nerves have been a focus of interest due to their direct relation to the CI performance $[6,13,17,20,23]$. ANF response according to the intra-cochlear electrode position has also been 
investigated in several studies, in which different and often contradictory conclusions were reported [24-29]. To reduce the spread of excitation and obtain a better pitch discrimination, an electrode position closer to the modiolus has been reported to decrease the threshold in which the first response on the ANF is obtained [24, 26]. On the other hand, some authors observed that the distance between the electrode and the modiolus did not affect, or affected minimally, this neural response threshold, or that its relation could not be clearly determined [27, 30]. Generally, a better speech perception of CI patients is obtained when the electrode is completely placed on the scala tympani [28, 29]. Lateral wall electrode arrays provide a higher probability to maintain this intra-cochlear position, whereas perimodiolar electrodes are likely to be misplaced, affecting directly the residual hearing preservation [28]. This issue is still an open discussion, yet it plays an important role when the electrode design needs to be chosen; thus, further studies are required.

The cochlea bears a tonotopic mapping, meaning that there is a frequency-position relation along the basilar membrane of the cochlea, following Greenwood's function [31]. This provides a frequency map that describes the related frequency ranges that a subpopulation of ANF bundles are sensitive to, according to their location in the cochlea. In particular, high frequencies are related to fibers at the base of the cochlea, and low frequencies to fibers at the apex. The optimal neural response caused by the electrode array is defined by its design, which determines the target frequency ranges. Thus, it is desired for each electrode inside the array to activate only a specific group of ANF bundles directly related to the frequency map by fitting the electrode parameters, such as stimulation amplitude or location, to obtain an optimal performance of the implant for the given patient. However, the characterization of the CI performance in terms of the computational ANF response has not been attempted before. Thus, it remains unclear whether the actual neural response can be used to provide an estimated measure for the further optimization of the implant performance.

In previous studies, we proposed an automatic framework for the generation of computational models of CI [32]. In this work, we further develop this model to compute the final neural response after CI. It includes a complete automatic in-silico assessment of the ANF activation on an improved computational CI model. The main objective of this article is to provide an automatic framework able to estimate and quantify the neural response evoked by the predicted intra-cochlear potential generated by the implant. This framework allows accounting for patient-specific anatomy, as well as surgical and electrical stimulation parameters that affect the surgery outcome [32].

Here, we present the development of the complete in-silico framework and the results obtained on specific CI scenarios. The methodology includes the generation of the finite element (FE) model (Section 2.1) and, following, the FE electrical simulations (Section 2.2). The obtained electric field causes a response of the ANF (Section 2.3) that provides information to assess the general intra- 
cochlear activation and quantify the overall CI performance on the specific patient (Section 2.4). The framework has been applied to evaluate the effects of (1) ANF degeneration (Section 3.1) and (2) the electrode intra-cochlear position (Section 3.2) on the activation thresholds according to the stimulation protocol employed.

\section{Methods}

\subsection{Finite element model}

Creation of a patient-specific CI model can be approached in different ways. A common approach is to obtain a simplified spiral 3D model created from a 2D cross-section of the cochlea and the electrode array $[19,22,26]$. In contrast, our model is based on high resolution anatomical images of the inner ear to create a detailed 3D model, rather than on geometrical simplifications. The step-by-step pipeline of the developed framework is shown in Figure 1., starting from the clinical CT of the patient (Figure 1.A), and resulting in the evaluation of the neural response (Figure 1.I). All elements of the CI computational model are created by applying our automatic framework for the generation of personalized meshes for FE modeling [32]. The framework includes a statistical shape model, an electrode array virtual insertion simulation algorithm, and the generation of a volumetric mesh of the inner ear. Anatomical $\mu \mathrm{CT}$ images were used to construct the highly detailed statistical model, process described in more detail in $[32,33]$. The creation of a statistical shape model of the inner ear allows us to generate virtual patients, by sampling the model. More importantly, it allows obtaining the patient-specific detailed anatomical cochlear shape from conventional low-resolution patient images, by aligning and fitting the model to the image (using a non-rigid B-spline image registration [34] (Figure 1.B).

Once the surface of the cochlea was obtained (Figure 1.B), the virtual insertion of the CI electrode was performed (Figure 1.C). This insertion was done by simulating the real scenario of the surgery using our surgical planning software, thus obtaining a realistic trajectory line of insertion, and then matching the centerline of the electrode array geometry with the trajectory line of the insertion [32, 35]. An electrode array design with 12 electrodes (19 contacts) was used based on Med-EL Flex ${ }^{28}$ design. The ANF around the cochlea were generated automatically according to the patient's cochlear shape, considering different anatomical landmarks, such as the organ of Corti and the beginning of the basilar membrane [32].

Generalized models of brain, scalp and skull were extracted from VHP CT Dataset (University of Iowa, Magnetic Resonance Research Facility) (Figure 1.D-E) and they were coupled with the cochlear surface and electrode array virtually inserted (Figure 1.F). The reference electrode was located on the scalp, according to its prescribed placement on implant housing. The simulation of this reference electrode has proved to have an important role on the current paths predicted by the 
electrical conduction model and consequently, on the neural excitation patterns $[15,36]$. All elements were merged and transformed into a single volumetric mesh (Figure 1.F), leading to a mesh free of intersections of approximately $2 \cdot 10^{6}$ tetrahedral elements. The mesh quality was checked to ensure a good convergence on the FE simulation [32]. Temporal bone conductivity was updated based on measurements in patients of electric field imaging (EFI) [6]. For more details on the implementation of the FE simulations, see [32].

Figure 1. Evaluation pipeline of the cochlear implant. From the clinical CT of the patient (A), the

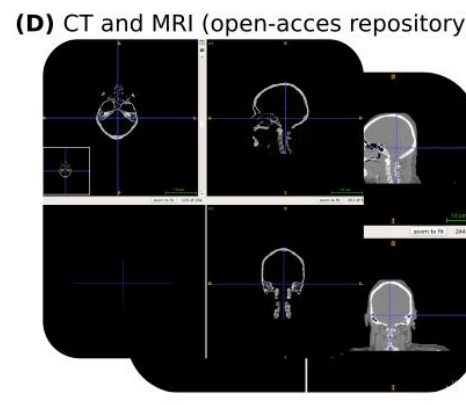

(E) Generalized head model

(A) Clinical CT

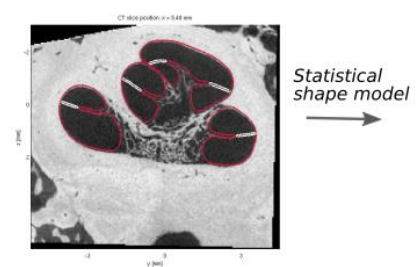

(B) Patient's cochlear surface

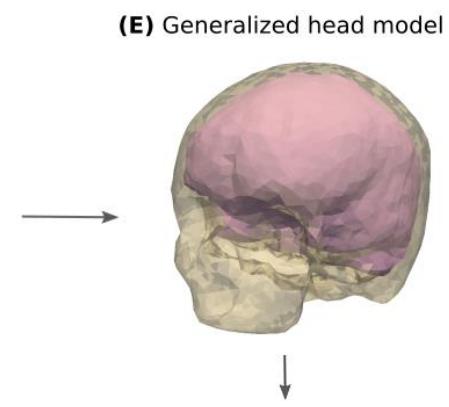

(F) Full head finite element mesh
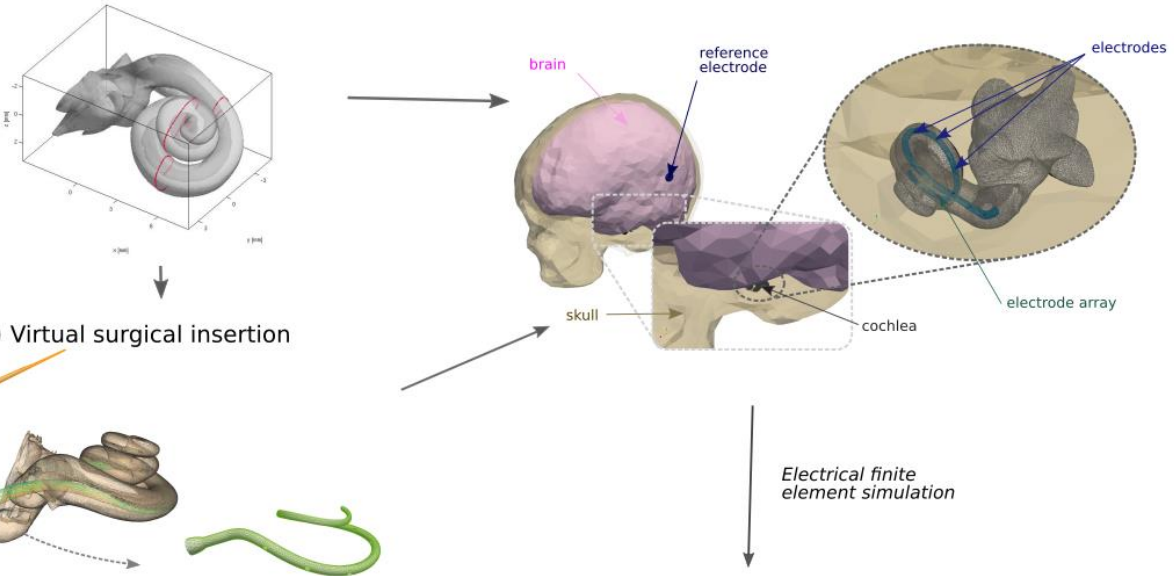

(I) Neural activation map

(H) Human neural fiber model
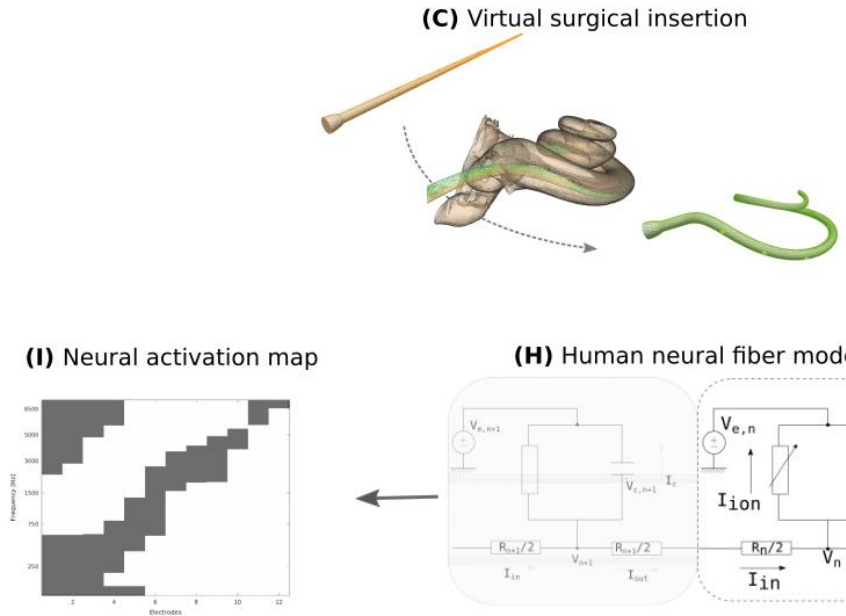

(G) Potential field
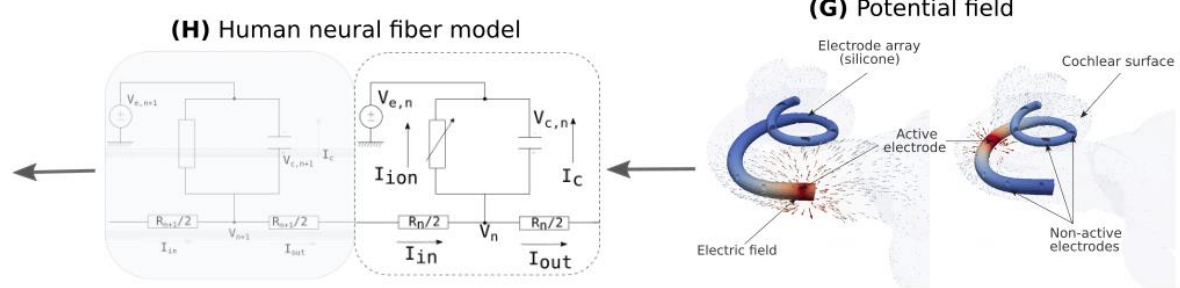

patient's cochlear surface (B) is extracted by the statistical shape model fitting procedure. A virtual surgical insertion (C) is performed with the desired surgical parameters. By segmentation from CT and MRI data from open-access repositories (D), a generalized head model (E) is created to be coupled with the patient's cochlear surface according to measurements extracted from MRI images of the cochlea localization. A full head tetrahedral finite element mesh $(F)$ is thus generated, allowing to perform computational electrical simulations. Then, the potential field created by the implant 
stimulation $(\mathrm{G})$ is obtained and, through the human auditory nerve fiber model $(\mathrm{H})$, the neural activation (I) is computed.

\subsection{FE simulation - electrical conduction model and stimulation parameters}

The electrical FE simulations were carried out considering an electrical conduction model and using the electrostatic solver of the open source Multiphysics software Elmer [37]. By solving the Poisson equation (Eq.1) in a quasi-static approximation regime, we obtained the electric field created by the CI stimulation (Figure 1.G):

$$
\nabla \cdot \sigma \nabla \phi=\frac{\partial \rho}{\partial t}
$$

where $\nabla$ is the gradient operator, $\rho$ is the electric conductivity, $\sigma$ the electric potential and $\phi$ the total current density. Different stimulation strategies can be set up in a CI, regarding the configuration of active electrodes. Monopolar, bipolar and tripolar protocols are the most common ones. Monopolar stimulation consists in configuring one intra-cochlear electrode as a source, while the reference is defined as an extra-cochlear electrode placed on the implant housing positioned on the bone [12]. This generates a higher voltage spread than other stimulation protocols [19, 38]. Bipolar stimulation places the reference on the intra-cochlear adjacent electrode, producing a more focused electric field, while tripolar stimulation uses two adjacent electrodes as reference. In this work, monopolar (MP) stimulation was used to assess the excitation spread in all ANF, considering the ground electrode in the implant housing located on the scalp (Figure 2).

The stimulus level delivered by the active electrode is directly related to the excitation current spread [17]. In previous studies, the amplitude of the stimulating current has been established in a broad range, which varies from $150 \mu \mathrm{A}$ to $5 \mathrm{~mA}[6,12,13,19,26]$. However, in the clinical practice a narrower range (from $200 \mu \mathrm{A}$ to $1 \mathrm{~mA}$ ) of stimulation amplitudes are commonly selected. In the current study, the maximum stimulation amplitude corresponds to the maximum one which is clinically used. In this way, it is possible to assess the maximum spread of excitation observed in the patient's cochlea (Figure 1.G), and thus, to evaluate accordingly the response of the ANF in the patient-specific case.

Since the model was considered purely resistive, the potential field obtained for different stimulation amplitudes was proportional to the one generated by a current of $1 \mathrm{~mA}$ (see Figure 4). By adapting this parameter, we determined the minimum amplitude needed to excite the desired range of nerve bundles. That is, we set the parameters to exclusively stimulate the target group of fibers that the specific electrode design aims to activate according to the tonotopic mapping of the cochlea. This allows evaluating, for instance, the cross-talk zones - activation of wrong frequencies. On those, the nerve fibers are stimulated by a higher voltage spread than the expected one, and consequently they 
have a non-desired neural response. The desired voltage spread can be defined as the one that exclusively activates the target nerve fiber bundles, avoiding any cross-talk (see Section 2.4 for further details).

To assess the response of the ANF, the temporal response of the stimulation needs to be computed in a transitory state. This stimulation is computed via the generation of pulses. Commonly, the MP strategy employs biphasic pulses in the clinical practice. Anodic pulses are defined when the return electrode is set to ground and the intra-cochlear electrode is the current source. On cathodic pulses the configuration is reversed, the return electrode on the scalp acts as current source and the intracochlear electrodes as ground. In our experiments, each pulse had a duration of $100 \mu$ s with a biphasic cathodic-first pulse. The potential field obtained (Figure 2) was used as an input to the nerve fiber model, needed to initiate the activation response [17-19, 21].

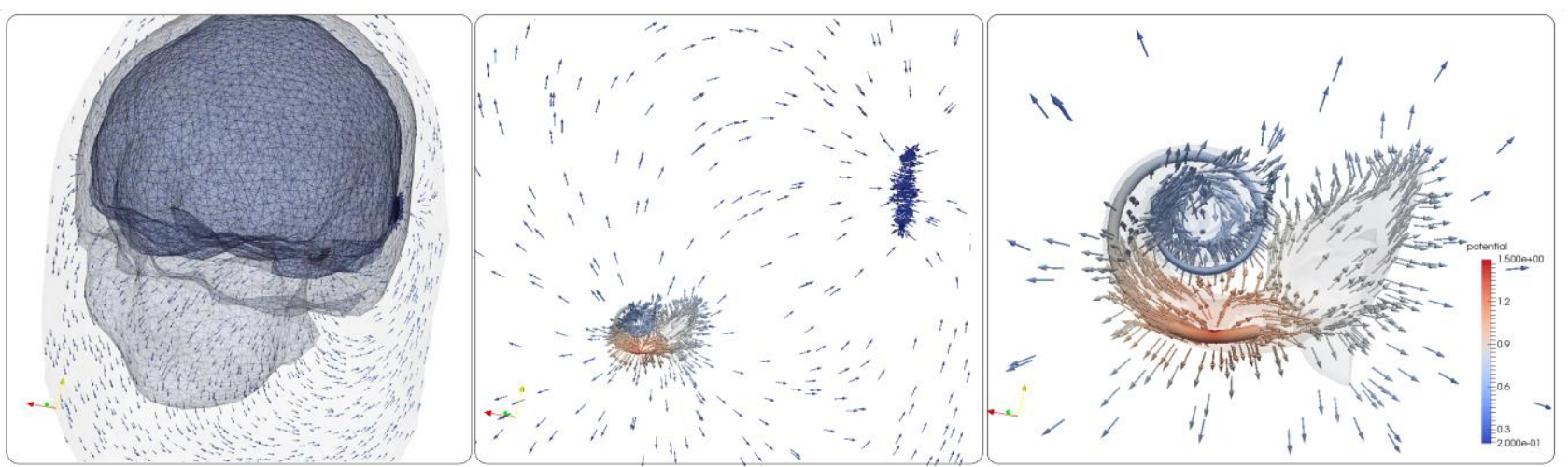

Figure 2. Potential field distribution. From left to right, zoom in captures of the whole model, that illustrate the potential field distribution from different perspectives when the monopolar stimulation strategy is used. In this example, the twelfth electrode (E12) is activated, while the reference electrode is set to ground.

\subsection{Human auditory nerve fiber model}

A Hodgkin-Huxley $(\mathrm{HH})$ compartment model was used to reproduce the membrane excitable behaviour of the neural cells [39]. The model provides the kinetics for activation and inactivation of the membrane ionic channels. Since the original implementation, proposed in the late 50 's, and based on the behaviour of the neural activation of a squid, several modifications have been proposed. Rattay et al. [18] presented a cable model of the human ANF, where each of the compartments that form the fiber was represented by an equivalent electrical circuit (see Figure 1.H). Each compartment has an external stimulation - extracellular potential - corresponding in our case to the outputs obtained from the FE simulation. The model accounts for the temperature effect found in humans and incorporated a speed up factor for the faster opening probabilities of channels. The multi-compartment model with human ANF morphology was implemented according to the description of [22], which also considered previous descriptions reported in [18]. We modelled 330 nerve fiber bundles spaced from 
each other $100 \mu \mathrm{m}$ along the organ of Corti of the inner ear. Along the human cochlea, there exist around 30.000 nerve fibers in the healthy cochlea and the mean length of the organ of Corti is 33.13 $\mathrm{mm}$ [40]. Thus, each fiber bundle computationally modelled contains 90 actual neural fibers, which retain enough frequency resolution along the cochlear duct [13].

The human ANF is composed by the peripheral axon, comprised by six internodes (which are removed in case of degeneration [18]), the pre-somatic region, the soma and the central axon with 16 internodes (see Figure 3). In total, a single fiber encompasses 47 compartments. The internodes of the peripheral and central processes have 40 and 80 layers of myelin, respectively, and the pre-somatic region soma, 4 layers $[18,22]$. The internode membranes have zero conductivity due to the insulating layers of myelin. The leakage conductance is inversely proportional to the layer of myelin: the higher the insulation of the myelin, the smaller the leakage.

Following Rattay's approach [18], the voltage potential of the neural cell membrane, $V_{m}$, can be expressed in terms of the capacitance, $C_{m}$, and the capacitive current, $I_{m}$, of the compartment as follows:

$$
\frac{d V_{m}}{d t}=I_{m} / C_{m}
$$

The input and output currents (Figure 1.H) of the current node are defined as:

$$
\begin{gathered}
I=\frac{\left(V_{m, n-1}+V_{e, n-1}\right)-\left(V_{m, n}+V_{e, n}\right)}{R_{n-1} / 2+R_{n} / 2} \\
I_{\text {out }}=\frac{\left(V_{m, n}+V_{e, n}\right)-\left(V_{m, n+1}+V_{e, n+1}\right)}{R_{n} / 2+R_{n+1} / 2}
\end{gathered}
$$

where $V_{e, n}$ is the extracellular potential on the nth compartment and $R_{n}$ the corresponding resistance. According to Kirchoff's law, expression (2) can be rewritten as:

$$
\frac{d V_{m}}{d t}=\left(-I_{\text {ion }}+I-I_{\text {out }}\right) / C_{m}
$$

where $I_{i o n}$ is the ionic current from the sodium, potassium and leakage channels (HH-model).

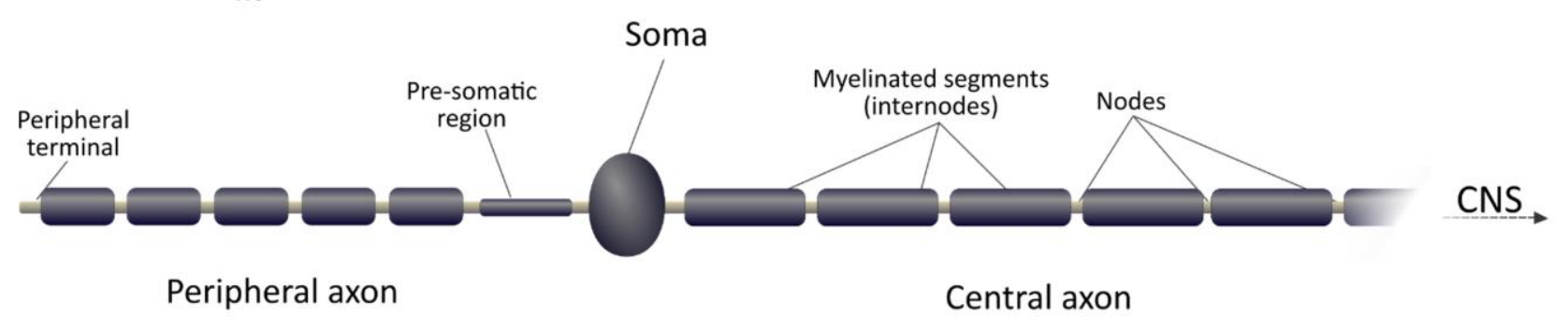

Figure 3. Illustration of an auditory nerve fiber. The impulse, or activation, created on the peripheral axon travels along the nerve fiber towards the central neural system (CNS) (Adapted from [22]). 


\subsection{Activation map and performance evaluation}

Evaluating the global stimulation pattern of a cochlear implant is not a straightforward process. In the clinical practice, there exist telemetry measurements, such as eCAP (evoked component action potential), that allow analysing the response of the ANF to an electrical stimulus. In computational models, a direct approach is to evaluate such a response assessing the action potential generation neural response to a stimulus- when delivering the electrical pulses. If this stimulus is able to create a spike that passes the soma, the spike is assumed to reach afterwards the central neural system, and therefore, the nerve fiber is considered to be excited.

This is the common approach to assess the neural response in CI models. However, the quantification of the outcome has not been addressed before due to the complexity of the real parameters that influence the intra-cochlear electrical potential generated by the CI. Such quantification is nonetheless of high relevance to provide information that can help pre-operatively on CI electrode array design decisions. Thus, we propose an evaluation measure to assess the implant neural activation performance. For this, we first define a local measure to evaluate each individual simulation in relation to the stimulating current intensity and the neural response obtained. Then, we obtain the optimal stimulation by defining the required stimulus intensity levels to cause ideal activation of the ANF with minimal cross-talk. Finally, from this optimized activation, a global performance measure is computed. In the following, we explain this procedure in detail.

Figure 4 Examples of activation maps (horizontal axis: stimulated electrode from the apex (E1) to the base (E12); vertical axis: frequency mapping of the cochlea). (A) Target frequency ranges according
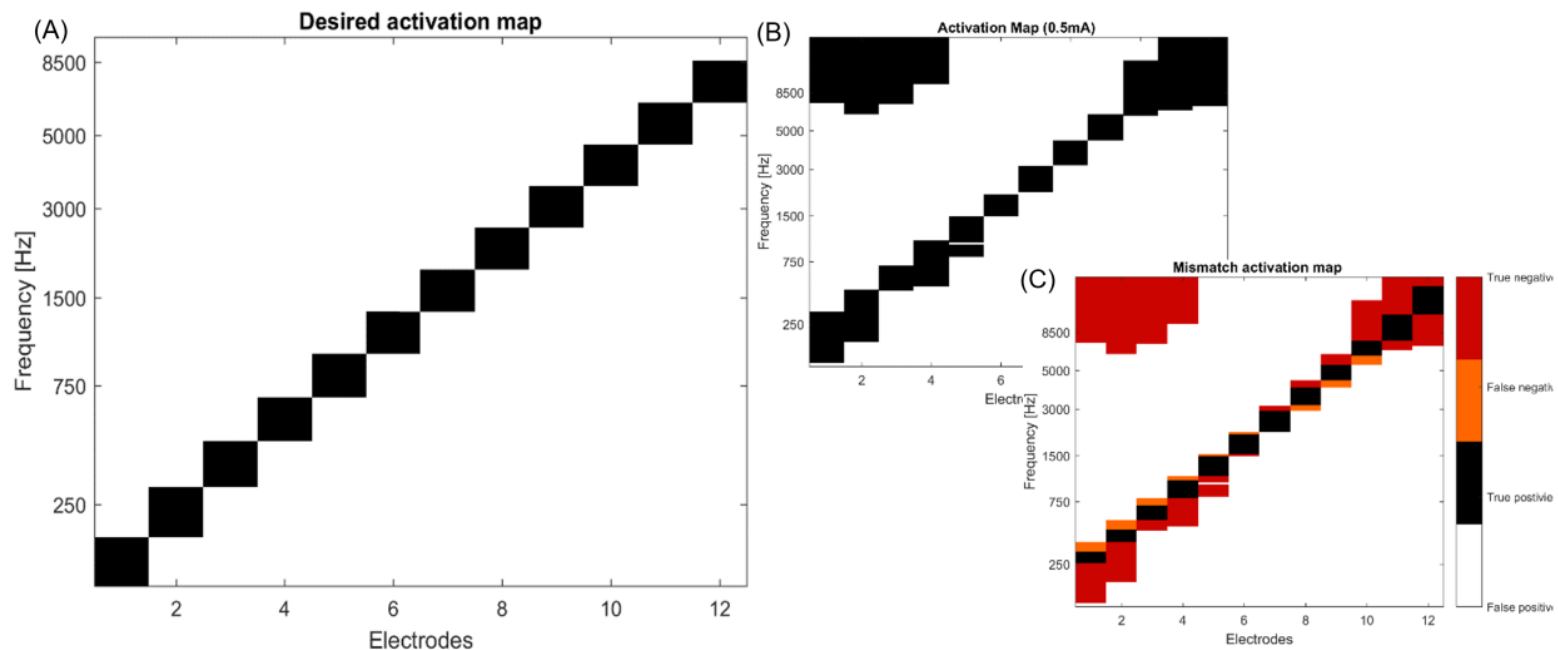

to the

electrode array design (Flex28, Med-EL). (B) Example of a patient-specific activation map for a monophasic (cathodic) stimulation protocol. (C) Mismatch activation map.

For the quantification of the neural response, a local activation map is computed. This map encompasses the neural response of the ANF of the cochlea for all 12 electrodes from the array (Figure 1.I). As described above, each electrode has its own target frequency bandwidth according to the 
cochlear tonotopic mapping and its location inside the cochlear duct. Figure 4.A shows an example of a desired activation map, stimulating selectively only the target ANF without any cross-talk. Using the proposed neural computational model, the obtained ANF activation is compared with the desired activation map (Figure 4.B). This leads to a mismatch activation map, which indicates the frequencies that have been properly or wrongly excited - true or false positives -or missed - false negatives (see Figure 4.C). Once the local activation map is computed, the neural response is optimized by defining the threshold level that produces the closest neural response to the desired one. To cover the range of stimulation amplitudes used on the clinical routine, a series of local activation maps for different stimulation amplitudes, from $200 \mu \mathrm{A}$ to $1 \mathrm{~mA}$, are obtained. The higher the amplitude, the wider the range of ANF activated.

The excitation of the ANF outside the desired bandwidth, known as cross-talk, plays an important role since it impairs hearing perception. For this reason, a local activation performance measure is important to quantify the mismatch for each electrode and intensity of the stimulation (Figure 5.B) and eventually, assess the global activation performance. This local activation measure $\delta_{i, e}$ quantifies the performance of the excitation profile, $f_{i, e}(x)$, obtained for the electrode $e$ and the impulse amplitude $i$. The strict excitation limits defined on Figure 4.A - and modelled for the electrode array design - are unrealistic in clinical practice, since the real voltage spread does not provide such a delimited field. Assuming this, each target bandwidth was modelled as a modified Gaussian distribution in such a way that locations that need to be excited are assigned positive values and otherwise are negative. Thus, this weighting function, $w_{e}(x)$, defines the ideal activation profile of the ANF. For each activation map, the weighting function corresponding to the different electrodes is applied (see Figure 5.B). The weighting function penalizes the excitation out of the ideal bandwidth by defining $w_{e}(x)>0$ for $x \in\left[\mu_{e}-\sigma_{e}, \mu_{e}+\sigma_{e}\right]$ and $w_{e}(x)<0$ otherwise, where $\mu_{e}$ is the central frequency target and $\sigma_{e}$ the bandwidth limits, both depending on the electrode. The local measure is defined by $\delta_{i, e}=\int_{0}^{\infty} f_{i, e}(x) \cdot w_{e}(x)$, where $\delta_{i, e} \in\left[p_{\min }, p_{\max }\right]$ with $p_{e, \min }=\int_{0}^{\infty} w_{e}(x)-$ $\int_{\mu_{e}-\sigma_{e}}^{\mu_{e}+\sigma_{e}} w_{e}(x)$ and $p_{e, \max }=\int_{\mu_{e}-\sigma_{e}}^{\mu_{e}+\sigma_{e}} w_{e}(x)$.

(A)

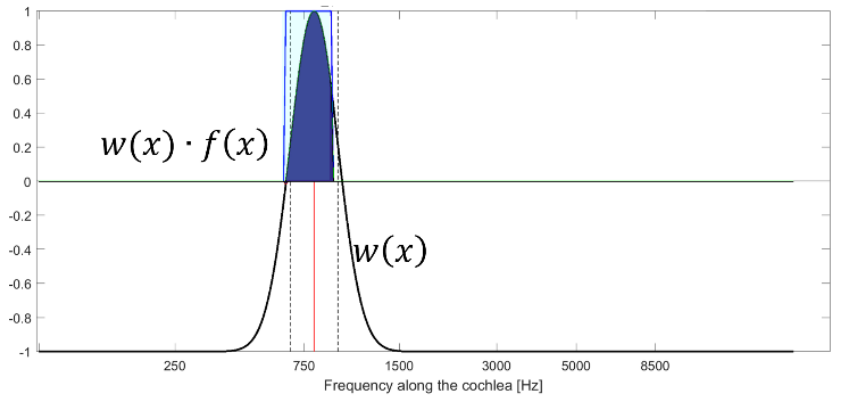

(B)

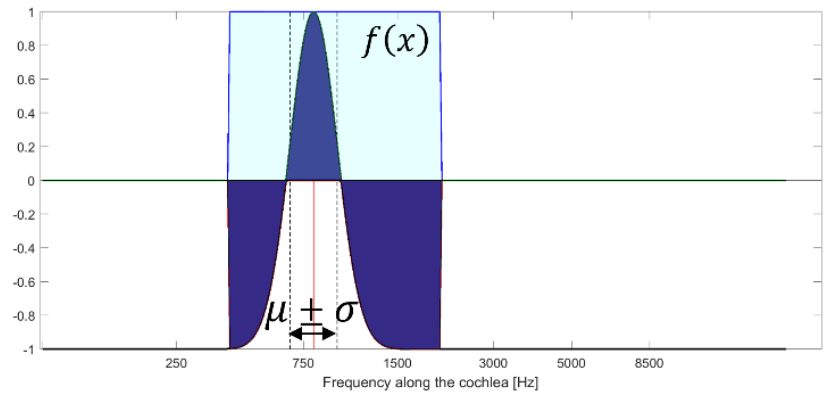

Figure 5 Local performance measure for the $5^{\text {th }}$ electrode when it is delivered an impulse of (A) 300 $\mu \mathrm{A}(\delta=91 \%)$ and $(\mathrm{B}) 800 \mu \mathrm{A}(\delta=47 \%))$. 
The midpoint of each electrode range $\left[p_{e, \min }, p_{e, \max }\right]$ was defined as $50 \%$ the performance, thus, mapping $p_{e, \min }$ and $p_{e, \max }, 0 \%$ and $100 \%$, respectively. Measures with no ANF response were considered as a zero value. The best local performance measure was chosen for each electrode which is equivalent to select the amplitude threshold which provides the closest excitation to the desired one. Then, a global activation map, composed by the excitation profiles of the identified thresholds, was computed (Figure 6.A). The mismatch activation map with a higher likelihood between the actual and the ideal ANF excitation was obtained (see Figure 4.C). Finally, the global performance is computed as $\Phi_{i}=\sum_{e=1}^{N} \delta_{i, e, \text { optim }} / N$, where $N$ is the number of electrodes in the array. This global measure and the optimized mismatch map enable to evaluate and compare easily the performance of CI simulation according to the electrode design, the patient and surgical parameters.
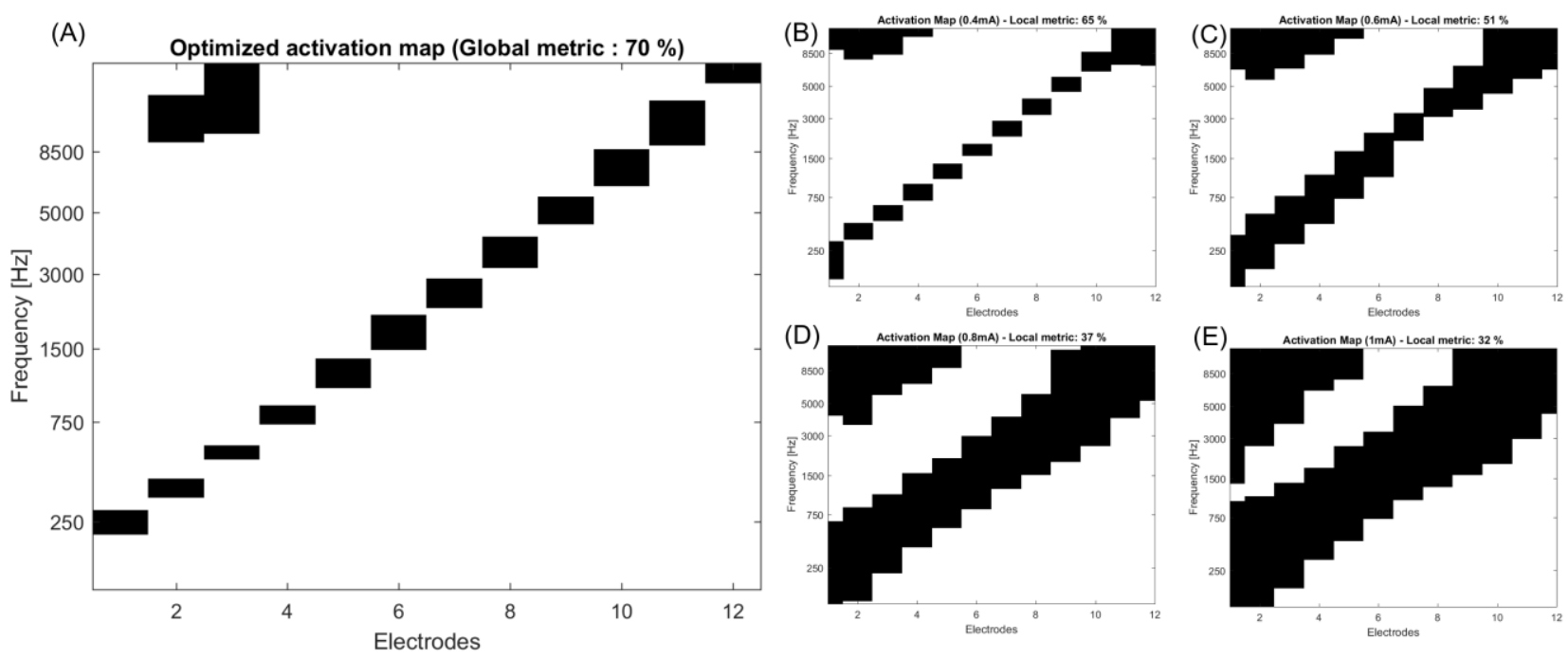

Figure 6. Performance measures and activation maps for (A) an optimized case $\left(\Phi_{i}=70 \%\right.$ and local ones for (B) $400 \mu \mathrm{A}\left(\Phi_{i}=65 \%\right.$, (C) $600 \mu \mathrm{A}\left(\Phi_{i}=51 \%\right.$,(D) $800 \mu \mathrm{A}\left(\Phi_{i}=37 \%\right.$ and (D) $1000 \mu \mathrm{A}$ $\left(\Phi_{i}=32 \%\right.$.

\section{Results}

The developed computational framework was employed to analyse the CI induced neural activation patterns on four different cases with distinct intra-cochlear position of the electrode array (lateral wall or perimodiolar) and ANF conditions (normal, degenerated). Firstly, positions of perimodiolar and lateral electrode array overlaid in a single cochlear 3D representation are presented in Figure 7A. Potential field distributions and the resulting ANF excitation are shown in Figures 7.B-C, respectively. These results correspond to the excitation profiles caused by activation of electrode 4 (E4), located at the medial-apical part of the cochlea and targeting the activation of ANF tuned to 
lower frequencies (central frequency $0.8 \mathrm{~Hz}$ ). The range of extracellular potential applied at the ANF model as input stimulus (Figure 7.C) was proportional to the one caused by $1 \mathrm{~mA}$, shown in Figure 7.B. The perimodiolar case shows a higher non-specific potential field on the basal part, from the beginning of insertion to the first half turn. Both activation profiles show that the higher the stimulus amplitude, the higher the cross-talk, as the electrode activates non-specifically also ANF tuned to higher frequencies (central frequency $12 \mathrm{kHz}$, and 12 and $4 \mathrm{kHz}$ for lateral wall and perimodiolar array, respectively), which are anatomically relatively close to the stimulating electrode due to the spiral shape of the cochlea. On the one hand, the perimodiolar position requires a higher stimulus intensity $(500 \mu \mathrm{A})$ to reach the whole desired frequency range of excitation (central target frequency $0.8 \mathrm{~Hz})$ while the lateral case requires lower intensity $(300 \mu \mathrm{A})$. On the other hand, the lateral wall configuration shows an overall higher voltage spread at lower stimulation intensities, and thus, less specific ANF excitation (Fig 7. C). Importantly, this spread is narrowed by decreasing the stimulus amplitude delivered, effect also seen in Figure 8.

Figure 7. (A) Models for perimodiolar and lateral wall position. Only cochlear and array meshes are displayed. (B) Potential filed distribution, E4 set as active source for $1 \mathrm{~mA}$. (C) Activation profile E4 within an amplitude range $[200 \mu \mathrm{A}, 1000 \mu \mathrm{A}]$.

(A)

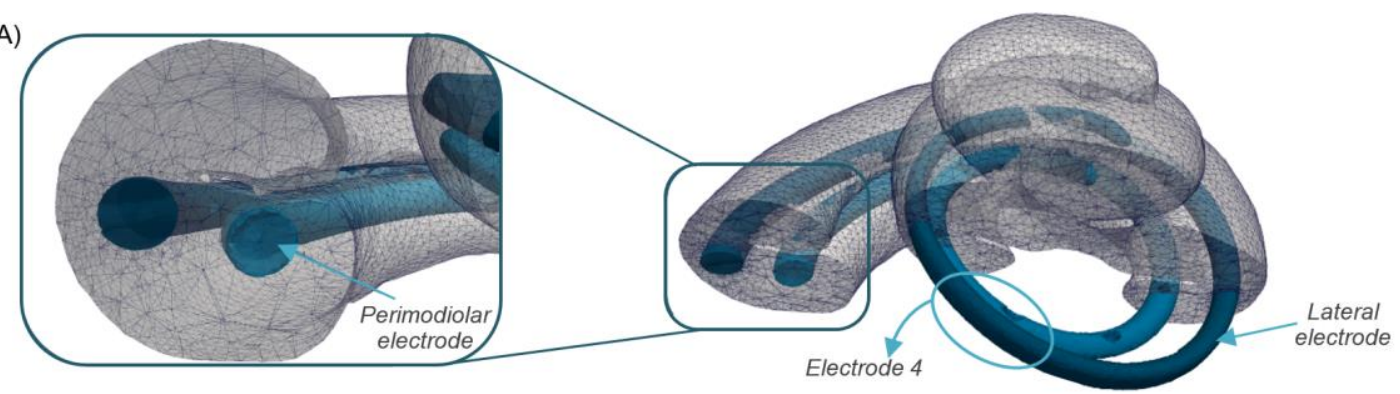

(B)

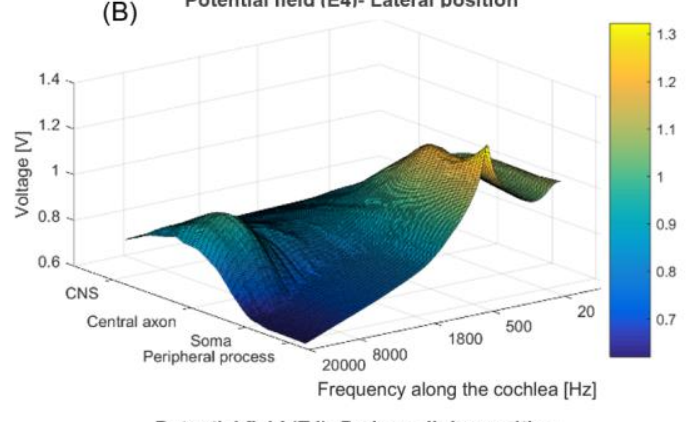

Potential field (E4)- Periomodiolar position

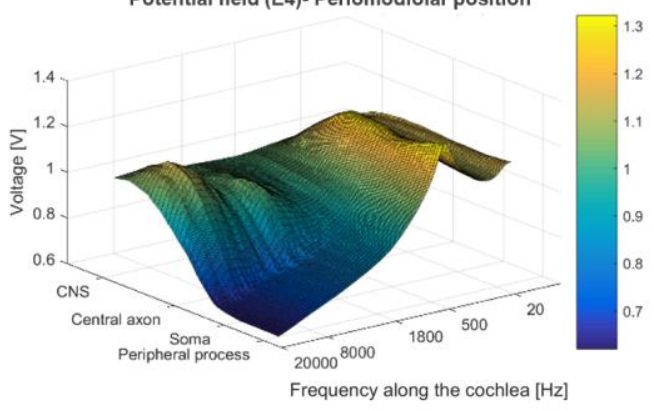

(C)

Results of the neural response of both intra-cochlear positions are presented in Figure 8. As mentioned above, the activation map contains information about the global ANF excitation caused by all 12 electrodes of the implant. To achieve that, activation profiles for each electrode of the array were calculated, similarly to Figure 7.C, and obtained results were transformed in a single activation map representing the activation of ANF with a given characteristic frequency evoked by stimulation of a given electrode with specific current amplitude. The presented results show the effect of the ANF degeneration (Figure 8.C and D), combined with both location approaches (Figure 8.A and B). As expected, in all cases a higher stimulation amplitude causes a wider, less specific, activation of the ANF. Consequently, as the intensity decreases, the range of excitation is narrowed. Cross-talk effect is present in all cases, although reduced in degenerated ones. The simulation indicates that if the stimulation amplitude is reduced to minimise the cross-talk, the target frequencies might be missed. This can be demonstrated in the lateral wall position, non-degenerated case, for the electrodes E1 and $\mathrm{E} 2$, which require an amplitude of $600 \mu \mathrm{A}$ to activate the target frequencies, from 150 to $700 \mathrm{~Hz}$, but the non-specific neural response already started at high frequencies for an amplitude of $300 \mu \mathrm{A}$ or below (Figure 8.A). This effect can be seen also in Figures 8.C-D for the degenerated cases. However, in these cases, higher intensities are required due to the degeneration of the ANF, thus a higher potential field is needed to obtain the desired excitation, which results in, as a consequence, the appearance of cross-talk. The perimodiolar approach for the case with ANF degeneration (Figure 8.D) does not cause cross-talk up to $500 \mu \mathrm{A}$. However, higher amplitudes from $600 \mu \mathrm{A}$ are required to excite the desired range of ANF. On the other hand, Figure 8.C shows that lateral wall electrode provides a lower activation threshold for the electrodes located on the base, while on the apex crosstalk starts from $400 \mu \mathrm{A}$.

Figure 9 presents dependence of local activation performance of the electrodes -directly related to the specificity- with amplitudes of the currents delivered. The trend in all four cases is a nonmonotonic decrease of the activation performance measure $\left(\delta_{i, e}\right)$ as the amplitude increases. In the degenerated cases (Figure 9.C-D), this measure is the highest for the threshold level, the smallest current for which non-zero neural response is obtained. On these, measure performances are generally decreased compared to the healthy case. This is also due to the penalization of the cross-talk effect. When there is a degeneration of the ANF, the optimal intensity is $100 \mu \mathrm{A}$ to $500 \mu \mathrm{A}$ higher than the healthy case (see values in Table 1). This causes cross-talk on the optimized stimulus amplitude (Figure 8.C-D). In the lateral wall position and non-degenerated case (Figure $8 \mathrm{~A}$ ), the local activation performance further decreases with increasing current amplitude below the threshold for electrodes E5 to E9, but does not change or slightly increases for other electrodes. This suggests that 
it is important to identify electrodes highly sensitive to stimulation current amplitude in terms of frequency specificity and not to overstimulate ANF with these, but rather to stay close to the threshold if specificity is the primary scope during the patient post-operative fitting. The electrodes in the perimodiolar and non-degenerated case (Figure 8.B) as well as the degenerated cases (Figure 8.C-D) do not seem to show such negative behaviour for higher currents than threshold ones, and larger current stimulation does not have any detrimental effect in terms of frequency selectivity.
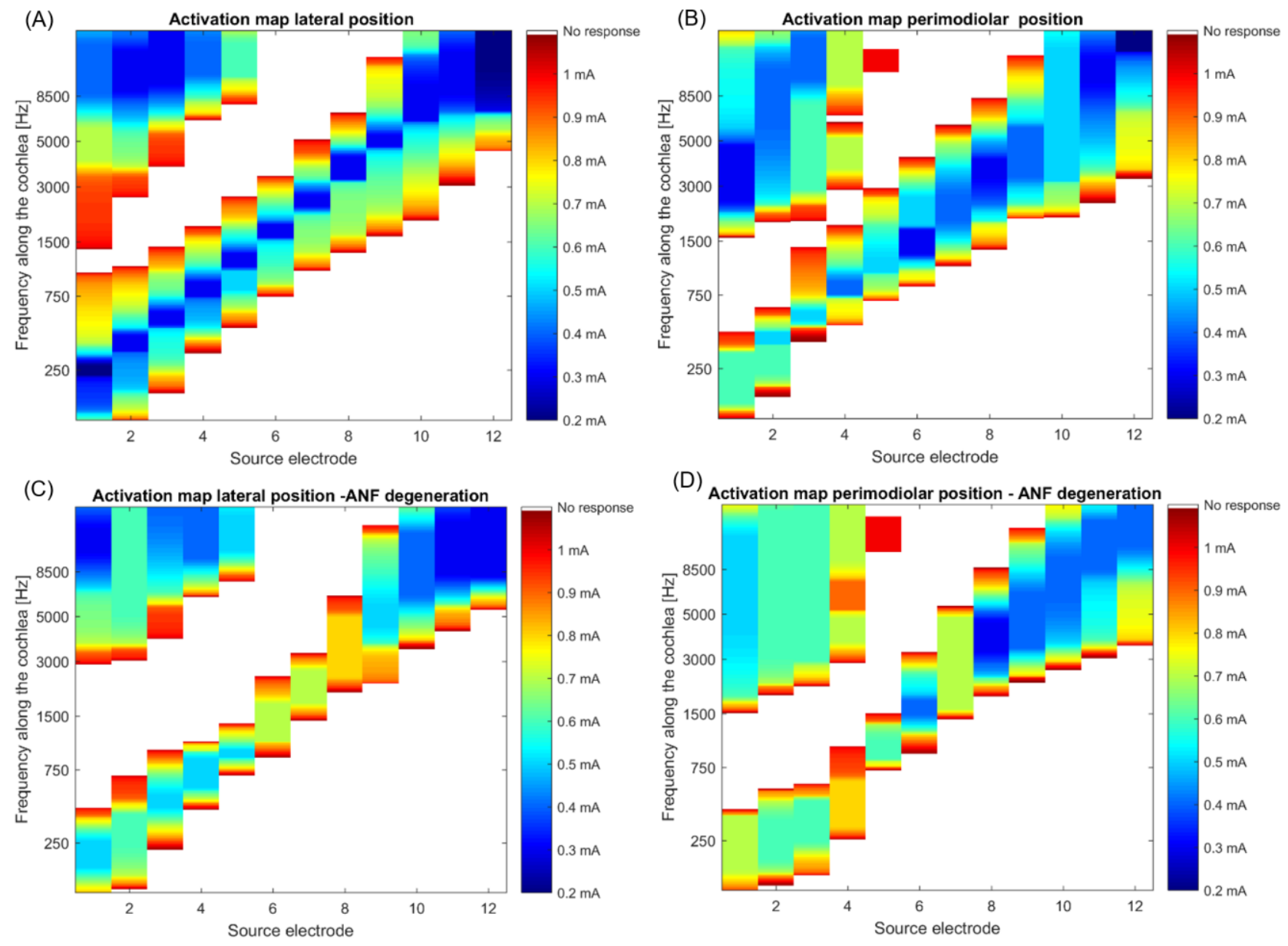

Figure 8. Activation maps for (A) lateral wall position and (B) periomodiolar position in healthy conditions; and lateral (C) and periomodiolar (D) for a case of degeneration of the ANF. Color scale shows the intensity of the stimulus. 
(A)

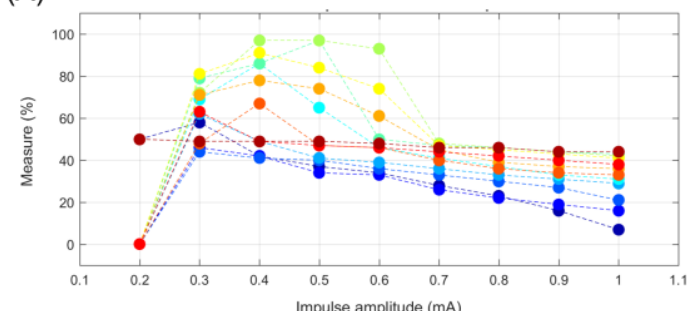

(C)

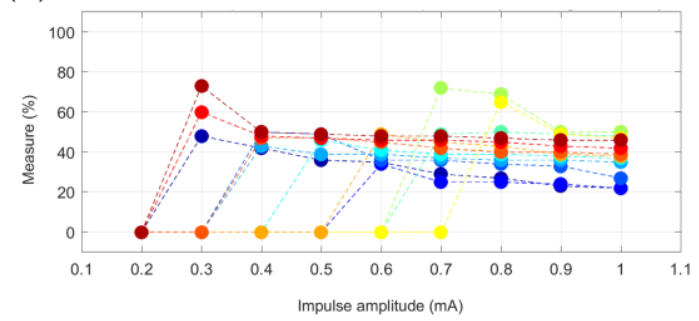

(B)

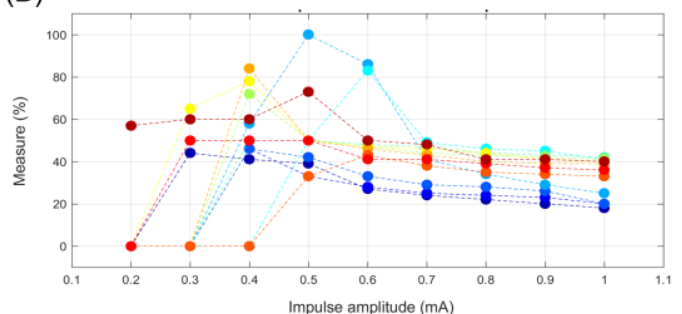

(D)

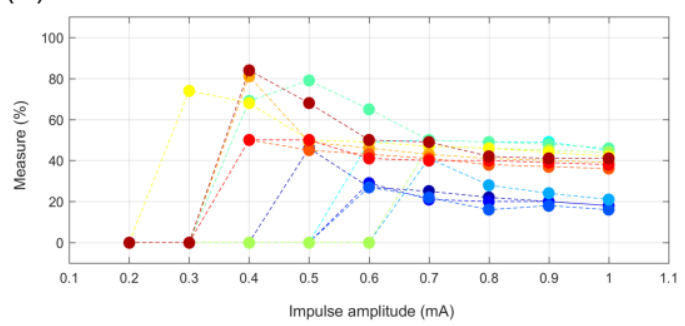

- E1

- E2

- E3

- E4

- E5

E6

E7

E8

- E9

- E10

- E11

- E12

Figure 9. Local performance at (A) lateral wall and (B) periomodiolar position in healthy conditions, and at lateral (C) and periomodiolar (D) position for a case of degeneration of the ANF.



Table 1. Optimized values ( $A_{\text {optim }}$ : Optimized amplitude (in $\left.\mathrm{mA}\right), \delta_{i, e}$ : Local performance measure).

The data from Figure 9 are summarized in Table 1. It shows that optimized amplitudes and their corresponding local performance values highly differ from one case to the other. In particular, degeneration of the ANF affects the CI performance by increasing the amplitude needed to achieve the excitation of the ideal frequency bandwidth. Some specific cases on the lateral electrode show decreases from $7 \%$ (E9) to $47 \%$ (E5) compared to the healthy case. For periomodiolar insertion, these values are decreased up to $79 \%$ (E4). The presented results show that the apical electrode (E1) provides higher spread, which provokes wider bandwidth activation. In case of neural degeneration, this excitation bandwidth is reduced and, in the same way, also the cross-talk at the base. This explains why for $300 \mu \mathrm{A}\left(\delta_{i, e}=73 \%\right)$ an improvement of a $23 \%$ in its performance is obtained, compared to the healthy case, for which the best measure is obtained at $200 \mu \mathrm{A}$ ( $\left.\delta_{i, e}=50 \%\right)$. However, this is not 
observed with periomodiolar electrodes, where higher intensities are required, reducing their performance. Some cases, such as electrodes located at the base, show a decrease of the amplitude with a slight improvement.

The optimized stimulation intensities for the four cases presented in Table 1 form each one a global activation map. By comparing to the target activation map (Figure 4.A), the mismatch map provides a fast visualization of the global CI performance (Figure 10). ANF correctly activated or missed are clearly identifiable by means of the provided color code. Generally, mismatch maps with high values indicate that the cross-talk remains. This effect is penalized in the proposed performance measure (Section 2.4). In particular, perimodiolar electrodes do not provide the assumed better performance in cases of ANF degeneration $\left(\Phi_{i}=50 \%\right.$ against $\Phi_{i}=52 \%$ for the lateral case), while lateral electrodes decrease significantly the cross-talk effect. This suggests that perimodiolar electrodes may be located too close of the soma of basal ANF, thus, creating a high cross-talk due to the high intensities (from 0.7 to $1 \mathrm{~mA}$ ) required to activate the target apical ANF. Figures 10.C-D suggest that intra-cochlear position needs to be carefully selected when neural degeneration occurs. High crosstalk is present in both cases, and this reduces the ideal frequency bandwidth effectively excited. We believe that this is due to the short distance from the source electrode located on the apical part to the soma of the basal ANF.
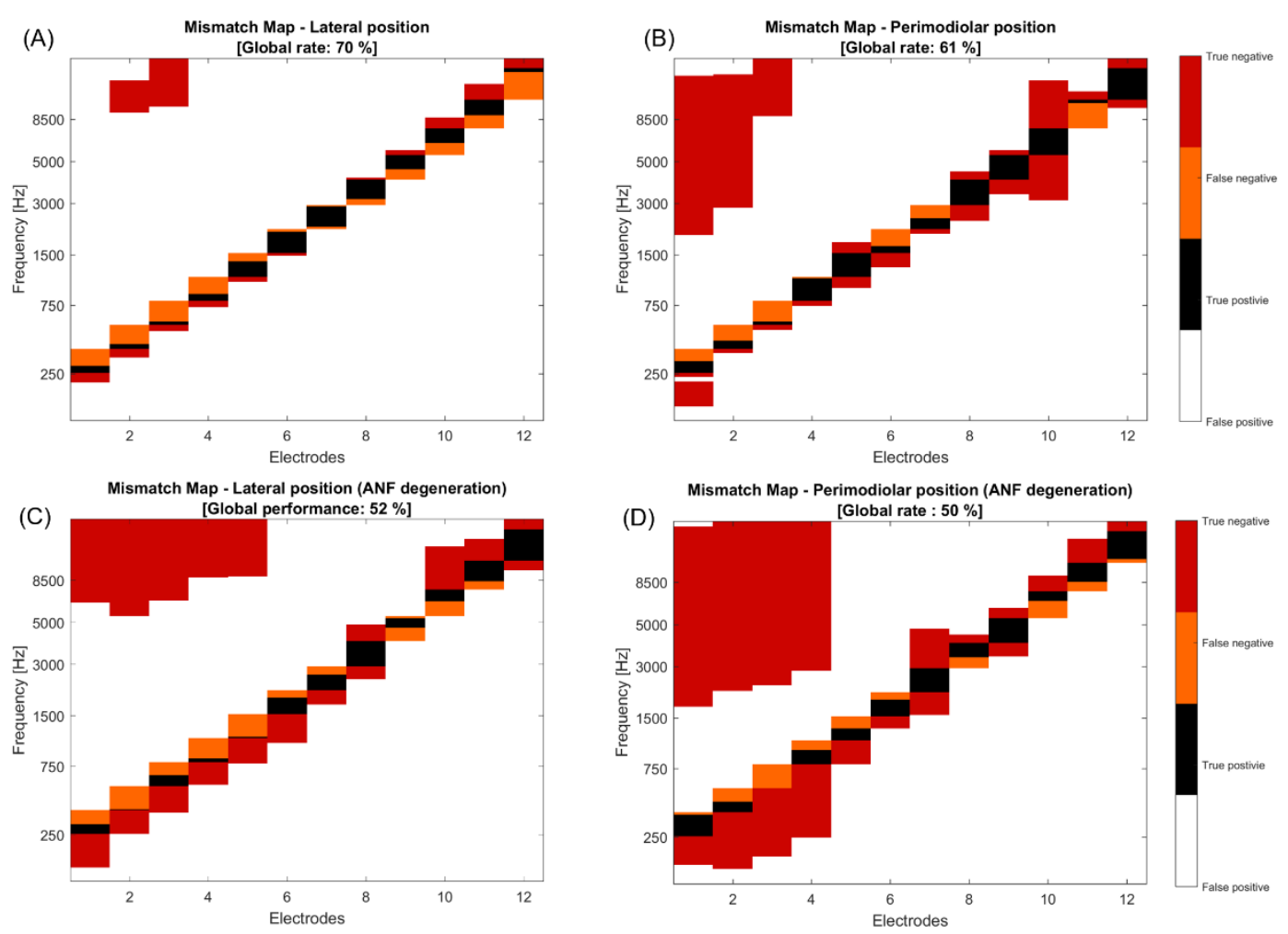

Figure 10. Mismatch optimized activation map. (A) Lateral wall position and (B) periomodiolar in healthy conditions. Lateral (C) and periomodiolar (D) for a case of degeneration of the ANF. 


\section{Discussion and conclusion}

We have presented a complete framework for the functional assessment of neural excitation with CI based on patient-specific factors, as well as on surgical and stimulation parameters. A new measure of CI performance is proposed to assess the resemblance of the actual stimulation with the target neural response. By evaluating the activation maps, we can set different stimulation parameters to optimize the activation and quantify the goodness of fit of the actual excitation provoked by the implant. The developed framework has been used to assess the neural response in different scenarios accounting for the electrode placement inside the cochlear scala tympani and the state of degeneration of the nerve fibers.

Initial results show that the framework provides reliable outcome results, which are consistent with previous findings in the literature and the clinical practice. Most importantly, similar potential fields were obtained emulating previously reported simulation parameters [15, 20]. Values obtained for the intra-cochlear voltage were in agreement with clinical measurements (within the range 0.19-0.35 V for a stimulus of $300 \mathrm{cu}$, equivalent to $300 \mu \mathrm{A}$ approximately). Normalizing the intra-cochlear voltage with the current injected, obtained impedance values were in concordance with EFI (Electric Field Image) measurements from patients reported on previous studies [41-43]. In addition, telemetry measurements, such as eCAP recordings, indicate that neural activation in ANF is initiated at $333 \pm 114$ cu [44]. This value, known as eCAP threshold, depends on the region of the cochlea where the stimulating electrode is located, and increases towards the base up to approximately $350 \mathrm{cu}$ [27, 44]. This effect is also seen in our simulations in the case of the lateral wall position, when the first ANF response is obtained at $300 \mu \mathrm{A}$, while in the apical region neural responses at lower amplitude are obtained. However, this is not observed in degenerated cases, in which the apical fibers require higher stimulus amplitudes. This suggests that ANF located at the apex are more affected by the degeneration of the peripheral process in terms of performance.

The proposed global and mismatch activation map provides a useful optimization tool for preoperative selection of optimal CI electrode array according to the patient anatomy and post-operative implant fitting of stimulation parameters for each patient. It allows to visualize clearly the general neural activation pattern of the implant and localize easily cross-talk effects. The presented results show a high presence of cross-talk in the apical region, which is common on CI patients. This effect is penalized in the proposed performance measure, which presents a clear and intuitive approach to quantify the performance of the patient-specific model. The quantification of the CI performance had not been attempted before, due to the complexity of the CI system and the interplay of the many 
factors that affect its performance. Our proposed measure seems to provide a reliable overview of this CI outcome.

The perimodiolar electrodes were originally designed to obtain the same performance with a lower stimulus threshold - compared to the threshold needed for straight electrodes inserted in a lateral wall position. However, the perimodiolar approach uses an electrode design that may cause severe internal trauma due to the penetration of the array and translocation to scala vestibuli, while having the same efficacy as lateral electrodes in terms of eCAP measurements [28]. There is still controversy about whether the electrode-modiolus distance has an influence on the CI outcome [20, 24, 26, 27, 29, 45]. Some authors determined that this distance does not make a difference on the neural activation, while the insertion depth plays an important role [27]. Others suggested the need of more research and to find an optimized design to preserve the structure and achieve the best outcome [46]. Our results show that lateral wall electrodes perform better for the healthy ANF case, while only slight differences are obtained in case of ANF degeneration. These results reveal that, for the cases under study, intracochlear electrode position plays a role on the ANF excitation. However, further studies are required to establish a correlation between these parameters and their performance. We believe that the computational tools presented in this work can be valuable to perform such studies.

Some limitations have been identified in the proposed computational framework. Although the generation of the model has been improved regarding previous works, nerve fiber geometry needs further improvements. Part of our current work goes towards more realistic anatomical shape of the ANF, improving spatial distribution along the cochlea and neuron density, similarly to [6]. Moreover, obtaining more accurate clinical data to validate computational results, such as CI telemetry measurements, is of prime importance to fully test computational results. However, the extraction of some experimental data involves clinical testing, which prolongs surgical time and patient anesthetization period. Such measurements should be attempted in the future to validate the obtained optimization results.

It would be interesting to use the proposed framework to assess patient-specific models retrospectively, where the electrode position can be obtained from post-operative data. In addition, including the generation of eCAP response in the model could be an important improvement to directly compare the computational ANF activation with clinical measurements. For this, further development of the temporal neural response is required to interpret the refractory effect seen in clinical eCAP telemetry. It is believed that electrode encapsulation affects eCAP measurements, since it creates an important change on electrode impedance. Thus, adding the electrode-tissue interface could provide more accurate results and consequently, predict long-term CI performance. 
Overall, the work presented here contributes with an automatic tool able to provide pre-operative predictions of the outcomes of the CI. This information can be used to optimize surgical and stimulation parameters for a given patient, or to assess the outcomes on a population of patients accounting for uncertain and variability in parameters such as cochlear shape anatomy [3] or tissue electrical conductivity [47]. This work is under development and is expected to fully cover the whole spectrum of parameters that can affect the outcome of the CI.

\section{Acknowledgements}

This work was financially supported by the European Commission (FP7 project number 304857, HEAR-EU) and the Spanish Ministry of Economy and Competitiveness under the Maria de Maeztu Units of Excellence Programme (MDM-2015-0502).

\section{References}

1. Erixon E, Högstorp H, Wadin K, Rask-Andersen H (2009) Variational Anatomy of the Human Cochlea. Otol Neurotol 30:14-22. doi: 10.1097/MAO.0b013e31818a08e8

2. Green K, Bhatt Y, Mawman D, O’Driscoll M, Saeed S, Ramsden R, Green M (2007) Predictors of audiological outcome following cochlear implantation in adults. Cochlear Implants Int 8:111. doi: $10.1002 /$ cii.326

3. Mangado N, Ceresa M, Dejea H, Kjer HM, Vera S, Paulsen RR, Fagertun J, Mistrik P, Piella G, Ballester MAG (2015) Monopolar Stimulation of the Implanted Cochlea: A Synthetic Population-Based Study. In: MICCAI 2015 Work. Clin. Image-Based Proced. Transl. Res. Med. Imaging. pp 96-103

4. Lee H-J, Giraud A-L, Kang E, Oh S-H, Kang H, Kim C-S, Lee DS (2006) Cortical Activity at Rest Predicts Cochlear Implantation Outcome. Cereb Cortex 17:909-917. doi: $10.1093 /$ cercor/bhl001

5. Giraud A-L, Lee H-J (2007) Predicting cochlear implant outcome from brain organisation in the deaf. Restor Neurol Neurosci 25:381-390. doi: Article

6. Kalkman RK, Briaire JJ, Frijns JHM (2015) Current focussing in cochlear implants: An analysis of neural recruitment in a computational model. Hear Res 322:89-98. doi: 10.1016/j.heares.2014.12.004

7. Rattay F, Leao RN, Felix H (2001) A model of the electrically excited human cochlear neuron. II. Influence of the three-dimensional cochlear structure on neural excitability. Hear Res 153:64-79. doi: 10.1016/S0378-5955(00)00257-4

8. Ceresa M, Mangado N, Andrews RJ, Gonzalez Ballester MA (2015) Computational models for predicting outcomes of neuroprosthesis implantation: the case of cochlear implants. Mol Neurobiol 52:934-941. doi: 10.1007/s12035-015-9257-4

9. Nogueira W, Schurzig D, Büchner A, Penninger RT, Würfel W (2016) Validation of a Cochlear Implant Patient-Specific Model of the Voltage Distribution in a Clinical Setting. Front Bioeng Biotechnol. doi: 10.3389/fbioe.2016.00084

10. Sibella F, Parazzini M, Pesatori A, Paglialonga A, Norgia M, Ravazzani P, Tognola G (2007) Modeling and Computation of Electric Potential Field Distribution Generated in Cochlear Tissues by Cochlear Implant Stimulations. In: 2007 3rd Int. IEEE/EMBS Conf. Neural Eng. IEEE, pp 506-509 
11. Zhu Z, Tang Q, Zeng F-GG, Guan T, Ye D (2012) Cochlear-implant spatial selectivity with monopolar, bipolar and tripolar stimulation. Hear Res 283:45-58. doi: 10.1016/j.heares.2011.11.005

12. Ceresa M, Mangado Lopez N, Dejea Velardo H, Carranza Herrezuelo N, Mistrik P, Kjer HM, Vera S, Paulsen RR, González Ballester MA (2014) Patient-specific simulation of implant placement and function for cochlear implantation Ssurgery planning. In: MICCAI. pp 49-56

13. Briaire JJ, Frijns JHM (2006) The consequences of neural degeneration regarding optimal cochlear implant position in scala tympani: A model approach. Hear Res 214:17-27. doi: 10.1016/j.heares.2006.01.015

14. Xiangming Zhang, Gan RZ (2011) A Comprehensive Model of Human Ear for Analysis of Implantable Hearing Devices. IEEE Trans Biomed Eng 58:3024-3027. doi: 10.1109/TBME.2011.2159714

15. Tran P, Sue A, Wong P, Li Q, Carter P (2015) Development of HEATHER for Cochlear Implant Stimulation Using a New Modeling Workflow. IEEE Trans Biomed Eng 62:728-735. doi: 10.1109/TBME.2014.2364297

16. Malherbe TK, Hanekom T, Hanekom JJ (2016) Constructing a three-dimensional electrical model of a living cochlear implant user's cochlea. Int J Numer Method Biomed Eng 32:e02751. doi: 10.1002/cnm.2751

17. Smit JEE, Hanekom T, Hanekom JJJ (2008) Predicting action potential characteristics of human auditory nerve fibres through modification of the Hodgkin-Huxley equations. S Afr J Sci 104:284-292.

18. Rattay F, Lutter P, Felix H (2001) A model of the electrically excited human cochlear neuron. Hear Res 153:43-63. doi: 10.1016/S0378-5955(00)00256-2

19. Malherbe TK, Hanekom T, Hanekom JJ (2013) Can subject-specific single-fibre electrically evoked auditory brainstem response data be predicted from a model? Med Eng Phys 35:926936. doi: 10.1016/j.medengphy.2012.09.001

20. Kalkman RK, Briaire JJ, Dekker DMT, Frijns JHM (2014) Place pitch versus electrode location in a realistic computational model of the implanted human cochlea. Hear Res 315:1024. doi: 10.1016/j.heares.2014.06.003

21. Frijns JHM, de Snoo SL, Schoonhoven R (1995) Potential distributions and neural excitation patterns in a rotationally symmetric model of the electrically stimulated cochlea. Hear Res 87:170-186. doi: 10.1016/0378-5955(95)00090-Q

22. Briaire JJ, Frijns JHM (2005) Unraveling the electrically evoked compound action potential. Hear Res 205:143-156. doi: 10.1016/j.heares.2005.03.020

23. Saba R, Elliott SJ, Wang S (2014) Modelling the effects of cochlear implant current focusing. Cochlear Implants Int 15:318-326. doi: 10.1179/1754762814Y.0000000081

24. Hughes ML, Abbas PJ (2006) Electrophysiologic channel interaction, electrode pitch ranking, and behavioral threshold in straight versus perimodiolar cochlear implant electrode arrays. $\mathbf{J}$ Acoust Soc Am 119:1538. doi: 10.1121/1.2164969

25. Long CJ, Holden TA, McClelland GH, Parkinson WS, Shelton C, Kelsall DC, Smith ZM (2014) Examining the electro-neural interface of cochlear implant users using psychophysics, CT scans, and speech understanding. JARO - J Assoc Res Otolaryngol 15:293-304. doi: 10.1007/s10162-013-0437-5

26. Kang S, Chwodhury T, Moon IJ, Hong SH, Yang H, Won JH, Woo J (2015) Effects of Electrode Position on Spatiotemporal Auditory Nerve Fiber Responses: A 3D Computational Model Study. Comput Math Methods Med 2015:1-13. doi: 10.1155/2015/934382

27. van der Beek FB, Briaire JJ, van der Marel KS, Verbist BM, Frijns JHM (2016) Intracochlear Position of Cochlear Implants Determined Using CT Scanning versus Fitting Levels: Higher Threshold Levels at Basal Turn. Audiol Neurotol 21:54-67. doi: 10.1159/000442513 
28. Venail F, Mura T, Akkari M, Mathiolon C, Menjot de Champfleur S, Piron JP, Sicard M, Sterkers-Artieres F, Mondain M, Uziel A (2015) Modeling of Auditory Neuron Response Thresholds with Cochlear Implants. Biomed Res Int 2015:1-10. doi: 10.1155/2015/394687

29. Wanna GB, Noble JH, Carlson ML, Gifford RH, Dietrich MS, Haynes DS, Dawant BM, Labadie RF (2014) Impact of electrode design and surgical approach on scalar location and cochlear implant outcomes. Laryngoscope 124:S1-S7. doi: 10.1002/lary.24728

30. Davis TJ, Zhang D, Gifford RH, Dawant BM, Labadie RF, Noble JH (2016) Relationship Between Electrode-to-Modiolus Distance and Current Levels for Adults With Cochlear Implants. Otol Neurotol 37:31-37. doi: 10.1097/MAO.0000000000000896

31. Greenwood DD (1990) A cochlear frequency-position function for several species--29 years later. J Acoust Soc Am 87:2592-605.

32. Mangado N, Ceresa M, Duchateu N, Kjer HM, Vera S, Dejea Velardo H, Mistrik P, R.Paulsen R, Fagertun J, Noially J, Piella G, González Ballester MAÁ, Duchateau N, Kjer HM, Vera S, Dejea Velardo H, Mistrik P, Paulsen RR, Fagertun J, Noailly J, Piella G, González Ballester MAÁ (2015) Automatic Model Generation Framework for Computational Simulation of Cochlear Implantation. Ann Biomed Eng 44:2453-2463. doi: 10.1007/s 10439-015-1541-y

33. Kjer HM, Vera S, Fagertun J, González Ballester MA, Paulsen R (2015) Predicting detailed inner ear anatomy from clinical pre-op CT. Proc. Comput. Assist. Radiol. Surg.

34. Kjer HM, Fagertun J, Vera S, Gil D, González Ballester MÁ, Paulsen RR (2016) Free-form image registration of human cochlear $\mu \mathrm{CT}$ data using skeleton similarity as anatomical prior. Pattern Recognit Lett 76:76-82. doi: 10.1016/j.patrec.2015.07.017

35. Duchateau N, Mangado N, Ceresa M, Mistrik P, Vera S, Ballester MAG (2015) Virtual cochlear electrode insertion via parallel transport frame. In: 2015 IEEE 12th Int. Symp. Biomed. Imaging. IEEE, pp 1398-1401

36. Wong P, George S, Tran P, Sue A, Carter P, Li Q (2016) Development and Validation of a HighFidelity Finite-Element Model of Monopolar Stimulation in the Implanted Guinea Pig Cochlea. IEEE Trans Biomed Eng 63:188-198. doi: 10.1109/TBME.2015.2480601

37. Ruokolainen J, Lyly M (2000) ELMER, a computational tool for PDEs--Application to vibroacoustics. CSC - News 12:30-32.

38. Stickney GS, Loizou PC, Mishra LN, Assmann PF, Shannon R V., Opie JM (2006) Effects of electrode design and configuration on channel interactions. Hear Res 211:33-45. doi: 10.1016/j.heares.2005.08.008

39. Hodgkin AL, Huxley AF (1952) A quantitative description of membrane current and its application to conduction and excitation in nerve. Bull Math Biol 117:500-544. doi: 10.1007/BF02459568

40. Stakhovskaya O, Sridhar D, Bonham BH, Leake P a. (2007) Frequency Map for the Human Cochlear Spiral Ganglion: Implications for Cochlear Implants. J Assoc Res Otolaryngol 8:220 233. doi: 10.1007/s10162-007-0076-9

41. Vanpoucke FJ, Boermans PB, Frijns JH (2012) Assessing the Placement of a Cochlear Electrode Array by Multidimensional Scaling. IEEE Trans Biomed Eng 59:307-310. doi: 10.1109/TBME.2011.2173198

42. Berenstein CK, Vanpoucke FJ, Mulder JJS, Mens LHM (2010) Electrical field imaging as a means to predict the loudness of monopolar and tripolar stimuli in cochlear implant patients. Hear Res 270:28-38. doi: 10.1016/j.heares.2010.10.001

43. Choi CTM, Wei-Dian Lai, Sih-Sian Lee (2006) A novel approach to compute the impedance matrix of a cochlear implant system incorporating an electrode-tissue interface based on finite element method. IEEE Trans Magn 42:1375-1378. doi: 10.1109/TMAG.2006.872461

44. Brill S, Müller J, Hagen R, Möltner A, Brockmeier S-J, Stark T, Helbig S, Maurer J, Zahnert T, Zierhofer C, Nopp P, Anderson I, Strahl S (2009) Site of cochlear stimulation and its effect on electrically evoked compound action potentials using the MED-EL standard electrode array. Biomed Eng Online 8:40. doi: 10.1186/1475-925X-8-40 
45. Saunders E, Cohen L, Aschendorff A, Shapiro Wand Knight M, Stecker M, Richter B, Waltzman S, Tykocinski M, Roland T, Laszig R, Cowan R (2002) Threshold, comfortable level and impedance changes as a function of electrode-modiolar distance. Ear Hear 23:28S--40S.

46. Gstoettner W, Kiefer J, Baumgartner W, Pok S, Peters S, Adunka O (2004) Hearing preservation in cochlear implantation for electric acoustic stimulation. Acta Otolaryngol 124:348-352.

47. Mangado N, Pons-Prats J, Ceresa M, Bugeda G, González Ballester MA (2016) Intracochlear potential prediction accountign for bone conductivity uncertainty. ECCOMAS Congr. 2016 